\title{
Les variables associées à l'attitude du personnel enseignant du secteur collégial vis-à-vis des étudiantes et étudiants présentant un trouble de santé mentale Variables related to the attitude of college teachers toward students with mental health disorders \\ Las variables asociadas a la actitud del personal docente en el sector colegial vis-à-vis los estudiantes que presentan un problema de salud mental
}

\section{Myreille St-Onge et Alexandre Lemyre}

Volume 44, numéro 1, printemps 2016

L'inclusion des étudiants en situation de handicap au postsecondaire : besoins, défis et enjeux

URI : https://id.erudit.org/iderudit/1036178ar

DOI : https://doi.org/10.7202/1036178ar

Aller au sommaire du numéro

\section{Éditeur(s)}

Association canadienne d'éducation de langue française

ISSN

1916-8659 (numérique)

Découvrir la revue

Citer cet article

St-Onge, M. \& Lemyre, A. (2016). Les variables associées à l'attitude du personnel enseignant du secteur collégial vis-à-vis des étudiantes et étudiants présentant un trouble de santé mentale. Éducation et francophonie, 44(1),

173-194. https://doi.org/10.7202/1036178ar

\section{Résumé de l'article}

Dans cet article, nous traitons des variables associées à l'attitude du personnel enseignant à l'égard d'étudiantes et étudiants présentant un trouble de santé mentale (TSM) et fréquentant seize cégeps au Québec. Ces variables regroupent le sexe, l'âge, l'expérience en enseignement et la source de connaissances sur les TSM. Le questionnaire évaluant l'attitude du personnel enseignant est constitué d'échelles du Student Mental Illness Awareness Survey et de trois échelles construites par l'équipe de recherche. Les résultats obtenus auprès de 232 enseignantes et enseignants sont présentés. Des analyses de variance univariées et des tests a posteriori ont été réalisées. Certaines variables sont associées à une attitude plus positive à l'égard de ces étudiantes et étudiants : être une femme, être plus jeune, mais aussi enseigner depuis plus longtemps et avoir acquis des connaissances sur les TSM lors d'une formation universitaire ou en milieu de travail. En contrepartie, l'acquisition de connaissances sur les TSM par l'intermédiaire des médias est associée à une attitude plus négative. Ces travaux appuient l'efficacité des formations visant à améliorer l'attitude du personnel enseignant à l'égard des étudiantes et étudiants ayant un TSM et ainsi faciliter leur parcours scolaire. Les résultats éclairent l'effet potentiellement négatif des médias sur les attitudes. Nous proposons deux stratégies étudiées chez d'autres populations afin de contrebalancer cet effet.
Tous droits réservés ( Association canadienne d'éducation de langue française, 2016
Ce document est protégé par la loi sur le droit d'auteur. L’utilisation des services d’Érudit (y compris la reproduction) est assujettie à sa politique d'utilisation que vous pouvez consulter en ligne. 


\section{Les variables associées à l'attitude du personnel enseignant du secteur collégial vis-à-vis des étudiantes et étudiants présentant un trouble de santé mentale*}

\section{Myreille ST-ONGE}

Université Laval, Québec, Canada

Alexandre LEMYRE

Université Laval, Québec, Canada

\section{RÉSUMÉ}

Dans cet article, nous traitons des variables associées à l'attitude du personnel enseignant à l'égard d'étudiantes et étudiants présentant un trouble de santé mentale (TSM) et fréquentant seize cégeps au Québec. Ces variables regroupent le sexe, l'âge, l'expérience en enseignement et la source de connaissances sur les TSM. Le questionnaire évaluant l'attitude du personnel enseignant est constitué d'échelles du Student Mental Illness Awareness Survey et de trois échelles construites par l'équipe

* Cette recherche a obtenu l'appui financier du ministère de l'Éducation, du Loisir et du Sport. 
de recherche. Les résultats obtenus auprès de 232 enseignantes et enseignants sont présentés. Des analyses de variance univariées et des tests a posteriori ont été réalisées. Certaines variables sont associées à une attitude plus positive à l'égard de ces étudiantes et étudiants : être une femme, être plus jeune, mais aussi enseigner depuis plus longtemps et avoir acquis des connaissances sur les TSM lors d'une formation universitaire ou en milieu de travail. En contrepartie, l'acquisition de connaissances sur les TSM par l'intermédiaire des médias est associée à une attitude plus négative. Ces travaux appuient l'efficacité des formations visant à améliorer l'attitude du personnel enseignant à l'égard des étudiantes et étudiants ayant un TSM et ainsi faciliter leur parcours scolaire. Les résultats éclairent l'effet potentiellement négatif des médias sur les attitudes. Nous proposons deux stratégies étudiées chez d'autres populations afin de contrebalancer cet effet.

\section{ABSTRACT}

\section{Variables related to the attitude of college teachers toward students with mental health disorders}

Myreille ST-ONGE

University Laval, Québec, Canada

Alexandre LEMYRE

University Laval, Québec, Canada

In this article, we discuss variables related to the attitude of teaching personnel toward students with mental health disorders (MHD) attending sixteen cégeps in Québec. These variables include gender, age, teaching experience and sources of knowledge about MHD. The questionnaire assessing the attitudes of teaching personnel is composed of the Student Mental Illness Awareness scale and three scales created by the research team. The results from 232 teachers are presented. Univariate a posteriori analyses were performed. Some variables are associated with a more positive attitude toward these students: being a woman, being younger, but also teaching for a longer time, and having acquired knowledge on MHD at university or at work. The acquisition of knowledge on MHD through the media is associated with a more negative attitude. These studies support the effectiveness of training that aims to improve teachers' attitudes toward students with an MHD, thus facilitating their academic journey. The results highlight the potentially negative effect of the media on attitudes. We propose two strategies studied in other populations to counterbalance this effect. 


\section{RESUMEN}

\section{Las variables asociadas a la actitud del personal docente en el sector colegial vis-à-vis los estudiantes que presentan un problema de salud mental}

Myreille ST-ONGE

Universidad Laval, Quebec, Canadá

Alexandre LEMYRE

Universidad Laval, Quebec, Canadá

En este artículo abordamos las variables asociadas con la actitud del cuerpo profesoral vis-à-vis los estudiantes que presentan un problema de salud mental (PSM) que frecuentan dieciséis colegios de Quebec. Dichas variables reagrupan el sexo, la edad, la experiencia docente y la fuente de sus conocimientos sobre los PSM. El cuestionario utilizado en la evaluación de la actitud del personal docente está constituido con las escalas del Student Mental Illness Awareness y tres escalas construidas por el equipo de investigación. Se presentan los resultados de 232 maestros y maestras. Se realizaron análisis de variancia univariada y a posteriori. Ciertas variables se encuentran asociadas con una actitud más positiva con respecto a dichos estudiantes: ser una mujer, ser más joven, así como enseñar desde hace mucho tiempo y haber adquirido conocimientos sobre los PSM durante su formación universitaria o en su medio de trabajo. La adquisición de conocimientos sobre los PSM gracias a los medios está asociada con una actitud más negativa. Esos trabajos se basan en la eficacia de las formaciones cuya finalidad es mejorar la actitud del personal docente con respecto a los estudiantes que presentan un PSM y así facilitar su itinerario escolar. Los resultados iluminan el efecto potencialmente negativo de los medios sobre las actitudes. Se proponen dos estrategias estudiadas entre otras poblaciones con el fin de contrabalancear dicho efecto. 


\section{Introduction}

Ces dernières années, les établissements d'enseignement postsecondaires ont connu une augmentation rapide du nombre d'étudiantes et d'étudiants présentant des incapacités requérant l'aide des services adaptés ou psychosociaux. Un certain pourcentage de ces étudiants présentent des troubles de santé mentale (TSM) dépression, troubles anxieux, etc. ${ }^{1}$. Il est reconnu que l'attitude du personnel enseignant est l'un des principaux contributeurs à la persévérance scolaire des étudiantes et étudiants présentant un TSM (Commission des droits de la personne et des droits de la jeunesse [CDPDJ], 2012; Rao, 2004; St-Onge, Tremblay et Garneau, 2009). En raison du rôle des enseignantes et des enseignants dans ce processus, il est primordial de s'intéresser aux variables susceptibles d'influencer leur attitude à l'égard des étudiantes et étudiants présentant un TSM.

\section{Le contexte de l'étude}

Dans l'optique de la présence accrue d'étudiantes et d'étudiants ayant un TSM dans les collèges, les enseignantes et enseignants sont appelés à interagir de plus en plus avec eux dans leurs classes. Un récent rapport de la Fédération nationale des enseignantes et des enseignants du Québec (FNEEQ, 2013) montre à quel point le personnel enseignant du secteur collégial doit revoir ses pratiques pour répondre aux demandes des services adaptés, tous types de déficiences ou d'incapacités confondus ${ }^{2}$. Nous avons fait ressortir, lors de la première phase de notre étude, que les principaux besoins du personnel enseignant avaient trait à la formation et à la sensibilisation aux TSM, mais aussi à l'accès à des personnes ressources pour les soutenir, les rassurer et les guider dans leurs actions auprès des étudiantes et des étudiants (St-Onge, Tremblay et Garneau, 2009) ${ }^{3}$. Bien que la fonction de conseiller ou conseillère de soutien aux services adaptés ait été créée depuis dans les collèges, les besoins de formation et de sensibilisation semblent encore criants (FNEEQ, 2013).

1. On se reportera au liminaire de ce numéro pour en savoir davantage sur le contexte d'émergence des étudiantes et des étudiants présentant des incapacités dans les universités et les cégeps québécois.

2. Dans cette étude, la définition attribuée aux TSM est exclusive aux comportements perturbateurs et aux crises dans les classes, c'est-à-dire «une déficience (sic) qui se manifeste par des comportements irritants ou des crises ayant donné lieu à une perturbation ou encore à une ou plusieurs interruptions de la prestation de cours» (FNEEQ, 2013, p. 57). Parmi les 748 répondants, 102 ont indiqué avoir été confrontés à de telles situations. Il faut noter ici le stigmate associé à cette définition qui envoie au personnel enseignant le message que la principale conséquence d'un TSM serait l'interruption de cours.

3. Cette étude comprenait trois phases. La première, menée auprès des répondantes locales, visait à établir l'état de développement du mécanisme d'aide offert aux étudiantes et aux étudiants ayant un TSM ainsi que les besoins des différents acteurs engagés auprès d'eux. La deuxième phase, qui fait l'objet du présent article, a consisté en la consultation en ligne du personnel enseignant. Enfin, la troisième phase, également une consultation en ligne, a été réalisée auprès de la population étudiante. 


\section{Le cadre conceptuel}

En nous appuyant sur les travaux de Marion Becker et ses collègues (2002), qui se sont intéressés aux attitudes du personnel enseignant d'une université de la Floride à l'égard des étudiantes et des étudiants ayant un TSM, nous avons évalué ces attitudes en contexte québécois. Il est reconnu que les attitudes prédisent les préjudices à l'égard d'une population donnée (Haddock et Zanna, 1993). Ces auteurs ont conceptualisé les attitudes selon trois dimensions: (1) cognitive - qui représente les connaissances que l'on possède sur un objet ou une population donnés; (2) affective - qui renvoie aux affects et aux sentiments que l'on a vis-à-vis de cette population; (3) comportementale - qui est la disposition à agir de façon favorable ou défavorable envers l'objet de l'attitude (Zanna et Rempel, dans Haddock et Zanna, 1993).

Le cadre conceptuel sur lequel est basée cette étude - le Modèle de développement humain et le Processus de production du handicap (MDH-PPH) de Fougeyrollas (2010) (voir la figure) -, qui inclut la notion d'attitudes (macrosystème), éclaire la réalité des étudiantes et étudiants ayant un TSM. Il stipule que les incapacités ne mènent pas systématiquement à une situation de handicap (p. ex. l'abandon des études). En effet, lorsque les incapacités interagissent avec des facteurs de protection, tels que l'attitude positive des enseignantes et enseignants appartenant au microsystème scolaire, il peut en résulter une pleine participation sociale dans ce domaine (la poursuite des études). 
Figure 1. Modèle de développement humain et Processus de production du handicap (MDH-PPH2) (Fougeyrollas, 2010)

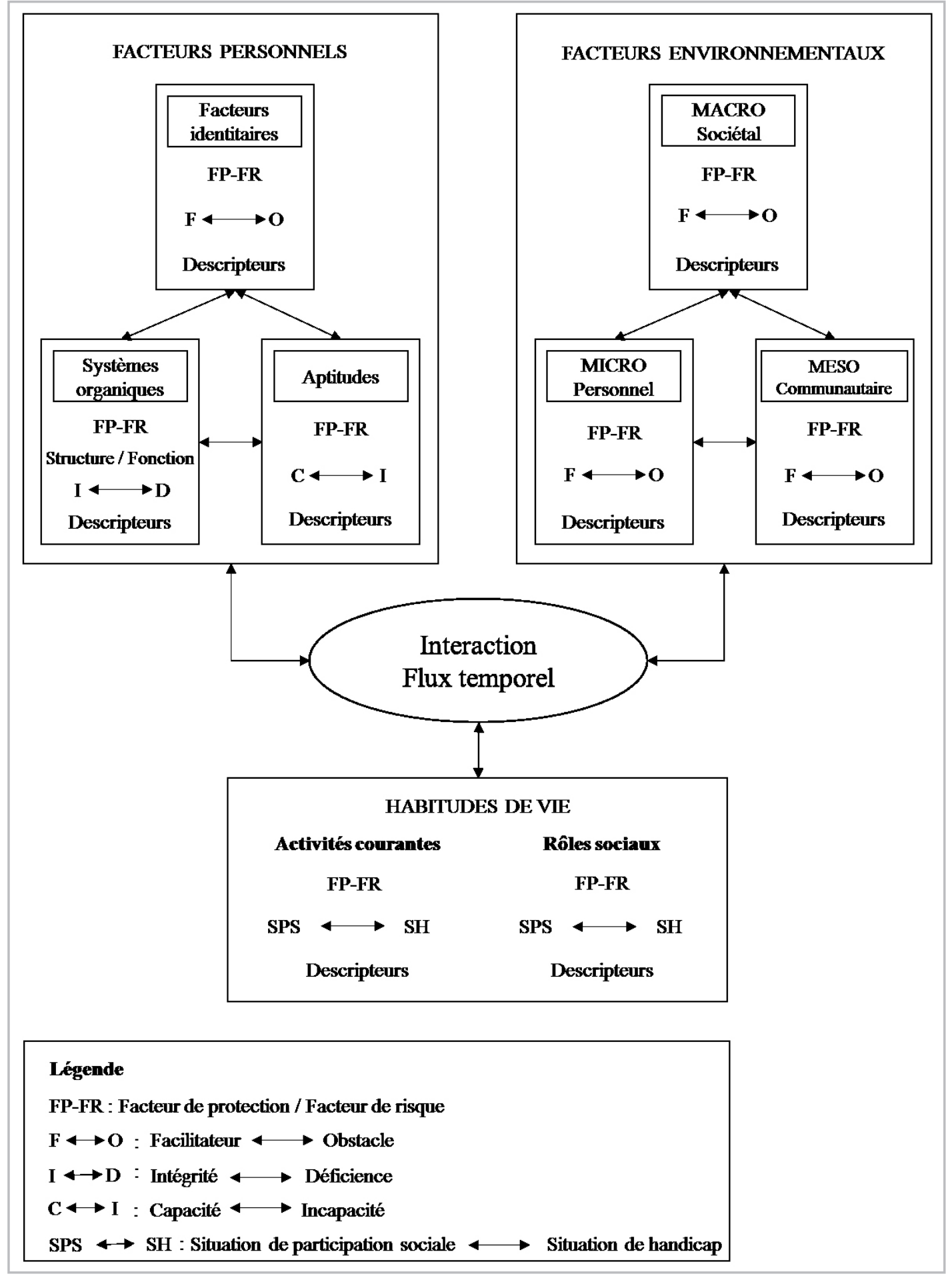


Selon Abric (2011), bien que les attitudes, positives ou négatives, soient résistantes au changement, elles évoluent en fonction de l'interaction qui se produit entre les individus, leur entourage social et le rang social qu'ils occupent. Le MDH-PPH permet justement de comprendre cette interaction dans l'optique de promouvoir la participation sociale optimale des personnes qui ont des différences sur le plan organique (déficiences) ou fonctionnel (incapacités). Dans ce modèle, une déficience est l'état d'un système organique altéré. Par exemple, dans le cas d'un étudiant atteint d'un trouble bipolaire, l'intégrité du rythme circadien du sommeil est perturbée (déficience) par des changements dans ses rythmes sociaux - la naissance d'un enfant par exemple - (Swartz, Frank et Frankel, 2008). En retour, cette perturbation peut provoquer la récurrence d'une phase hypomaniaque ou maniaque, accompagnée de comportements à risque (incapacités). Ces incapacités, en interaction avec des attitudes négatives de ses professeurs, sont susceptibles de le conduire à des relations interpersonnelles difficiles avec le milieu scolaire, ce qui peut conduire à l'abandon des études (situations de handicap). Nous ne pouvons donc déduire les situations de handicap sur l'unique base des limitations fonctionnelles (Fougeyrollas et al., 1998, p. 15). C'est en tenant compte des obstacles que cet étudiant rencontre (p. ex. les attitudes négatives de ses collègues ou de ses enseignantes et enseignants) ou des facilitateurs (p. ex. une bonne coordination des services au regard des interventions) que l'on sera en mesure d'évaluer ses habitudes de vie liées aux études (participation sociale ou situation de handicap). La réponse de l'entourage scolaire est donc essentielle à prendre en compte pour déterminer si un étudiant ayant un TSM est en situation de handicap ou non.

L'objet du présent article est d'exposer les différences quant aux attitudes des enseignants et des enseignantes et à leurs besoins en fonction de quatre caractéristiques, c'est-à-dire l'âge, le sexe, l'expérience en enseignement et la source de connaissances sur les TSM - formation universitaire, formation en milieu de travail, médias. Les attitudes d'ordre cognitif, affectif et comportemental sont évaluées par les variables suivantes: (a) la confiance en sa capacité à repérer les étudiantes et étudiants ayant un TSM et à les convaincre d'obtenir de l'aide, (b) la crainte et la distance sociale devant les étudiantes et étudiants présentant un TSM, (c) la perception des mesures offertes par les services adaptés et les services psychosociaux du collège, (d) les mesures offertes dans la classe pour les aider. Enfin, nous avons évalué les besoins que le personnel enseignant ressentait par rapport à ces étudiantes et étudiants. Selon Brockelman, Chadsey et Loeb (2006), les attributs personnels et les formations propres aux TSM sont susceptibles de prédire l'attitude des enseignants à l'égard des étudiants présentant un TSM.

\section{Les attributs personnels}

\section{Les hommes et les femmes}

Rao (2004) a recensé neuf études évaluant les différences entre l'attitude des enseignantes et des enseignants vis-à-vis des étudiantes et étudiants présentant une incapacité. Cinq études montrent une attitude plus positive chez les femmes, alors que quatre ne rapportent pas de différence entre les sexes. Depuis, de nouvelles 
études ont rapporté une attitude plus positive chez les femmes (Abu-Hamour, 2013; Hammel, 2010; Hawkins, 2013; Leyser et Greenberger, 2008; Murray, Wren et Keys, 2008). Toutefois, d'autres auteurs n'ont pas observé une telle différence (Becker, Martin, Wajeeh, Ward et Shern, 2002; Kraska, 2003; Lombardi, 2010). Becker et ses collaborateurs (2002) soulignent que les femmes ont davantage confiance en leur capacité à partager leurs inquiétudes avec les étudiantes et étudiants présentant des indices de TSM et à convaincre ces derniers de chercher de l'aide.

\section{L'âge}

Rao (2004) a également recensé cinq études évaluant le lien entre l'âge du personnel enseignant et leur attitude vis-à-vis des étudiantes et étudiants présentant une incapacité. Toutes rapportent des résultats non significatifs. De nouvelles recherches ont aussi échoué à trouver une association entre ces variables (Leyser et Greenberger, 2008; Kraska, 2003; Worthy, 2014). Toutefois, Abu-Hamour (2013) rapporte que le personnel enseignant ayant une à cinq années d'expérience a une attitude plus favorable quant à l'intégration aux études supérieures des étudiantes et étudiants présentant une incapacité. De plus, selon Becker et al. (2002), les enseignantes et enseignants les plus jeunes dirigent davantage d'étudiantes et d'étudiants vers le centre de consultation de leur établissement scolaire. Finalement, Brockelman et al. (2006) n'ont pas observé d'association entre l'âge du personnel enseignant et la facilité à travailler auprès des étudiantes et étudiants présentant un TSM; toutefois, l'âge est positivement associé à la confiance à travailler avec eux.

\section{L'expérience en enseignement}

Trois études (Leyser et Greenberger, 2008; Kraska, 2003; Worthy, 2014) n’ont observé aucune association entre l'expérience en enseignement et l'attitude à l'égard des étudiantes et des étudiants ayant des incapacités. Toutefois, Becker et al. (2002) rapportent que l'expérience en enseignement est positivement associée au nombre de consultations demandées pour les étudiantes et les étudiants à l'intérieur et à l'extérieur de l'établissement scolaire, ainsi qu'au nombre de mesures offertes.

\section{Les sources de connaissances}

Le personnel enseignant ayant bénéficié d'une formation sur les incapacités ateliers, classes - ou s'étant documentés - livres ou articles, journaux, Internet, etc. présente une attitude plus positive et davantage d'ouverture à offrir des mesures que les autres enseignantes et enseignants (Hammel, 2010; Leyser et Greenberger, 2008; Lombardi, 2010; Murray, Lombardi, Wren et Keys, 2009). Ces derniers auteurs précisent que ceux qui ont participé à un atelier ou qui ont suivi un cours ont une meilleure connaissance générale des incapacités que les enseignantes et les enseignants qui se sont seulement documentés. 


\section{La méthodologie \\ Le recrutement}

Un échantillon stratifié de seize cégeps de l'Est et de l'Ouest du Québec a été réalisé en considérant le milieu géographique (pôles urbains ou régions périphériques), le nombre d'étudiantes et d'étudiants et le ratio entre ce nombre et les ressources spécialisées dans chaque cégep. L'échantillon final est composé de quatorze cégeps francophones et de deux cégeps anglophones ${ }^{4}$. Nous avons recensé 3766 enseignantes et enseignants travaillant à temps plein ou à temps partiel dans l'ensemble de ces 16 cégeps. Une lettre contenant des informations sur la recherche et le lien conduisant au questionnaire a été expédiée par voie électronique à chacun d'eux. Quatre relances ont été effectuées.

\section{L'admissibilité des participantes et participants}

Afin de vérifier l'admissibilité des participantes et participants, nous avons utilisé l'échelle d'observation des comportements en classe (Becker et al., 2002). Nous posions la question suivante: «Dans une classe habituelle, dans quelle mesure avezvous observé chez des étudiants les comportements suivants qui peuvent être des symptômes d'un trouble mental ${ }^{5}$ ?» Cette échelle compte 12 énoncés en 4 points allant de jamais (1) à souvent (4). Des exemples d'énoncés sont: «Des absences répétées, des retards, des départs avant la fin du cours»; «Un retrait, une diminution de la cordialité»; "Des gestes ou des comportements étranges ou exagérés.» Les enseignantes et enseignants qui n'avaient observé aucun des comportements décrits dans cette échelle au cours des trois dernières années n'avaient pas à remplir le questionnaire.

\section{Les mesures}

L'échelle de la confiance pour identifier les étudiantes et étudiants ayant un TM et en sa capacité à convaincre un étudiant d'aller chercher de l'aide (Becker et al., 2002) comporte deux construits distincts expliquant $55,2 \%$ de la variance, pour un total de 10 énoncés en 4 points allant de pas du tout d'accord (1) à tout à fait d'accord (4) (St-Onge et Tremblay, 2009) ${ }^{6}$. Le premier construit - la capacité de détection d'un TM $(\alpha=0,83)$ - compte cinq énoncés, dont voici des exemples : «Un étudiant montrant des signes de stress peut avoir un trouble mental», «Un étudiant qui utilise un langage vague ou inusité peut avoir un trouble mental.» Le second construit - la confiance en sa capacité à convaincre un étudiant d'aller chercher de l'aide $(\alpha=0,71)-$

4. Cet échantillonnage a été réalisé en étroite collaboration avec la Direction des affaires étudiantes universitaires et collégiales (DAEUC) sous la supervision de madame Hélène Bonnelli, alors responsable du suivi de cette étude au MELS.

5. Nous avons utilisé, à l'instar des auteurs du rapport Aspect humain de la santé mentale et de la maladie mentale au Canada (Gouvernement du Canada, 2006, p. 2), le terme «trouble mental» dans notre étude. Ce terme renvoie à des manifestations qui interfèrent de manière significative avec les capacités cognitives, émotives ou sociales d'une personne.

6. Il est à noter que des analyses factorielles (varimax) avec rotation orthogonale, ainsi que des calculs de coefficients de cohérence interne, ont été réalisés pour chacune des échelles dans le cadre de cette recherche. Pour plus de détails, on peut obtenir le rapport sur les analyses de validité en s'adressant à la première auteure: Myreille.St-0nge@svs.ulaval.ca. 
compte cinq énoncés, par exemple: «J'essaierais de convaincre un étudiant ayant un trouble mental de chercher de l'aide à l'extérieur du collège», «Je suis capable de discuter de mes inquiétudes envers un étudiant sur ses signes de trouble mental que j'ai observés».

L'échelle de crainte et de distance sociale devant les étudiantes et étudiants présentant un TM (Becker et al., 2002) comporte deux construits expliquant 42,3\% de la variance, pour un total de 16 énoncés en 4 points allant de pas du tout d'accord (1) à tout à fait d'accord (4) (St-Onge et Tremblay, 2009). Le premier construit - les attitudes négatives à l'égard des TM $(\alpha=0,85)$ - comporte 12 énoncés, dont voici des exemples: «L'état mental d'une personne affecte négativement mon attitude envers elle», "On ne peut faire confiance à une personne ayant un trouble mental.» Le second construit - la connaissance et la compréhension des troubles $(\alpha=0,67)$ - comporte quatre énoncés, par exemple: «Je suis habituellement en mesure de dire quand des étudiants qui fréquentent mes cours ont un trouble mental», «Je comprends les causes des troubles mentaux».

Trois échelles ont été construites à partir des informations obtenues lors de la première phase de l'étude auprès des répondantes locales et du personnel professionnel de la relation d'aide des seize cégeps, et lors d'un groupe de discussion auprès de psychologues de trois cégeps des régions de Québec et de la ChaudièreAppalaches. Un prétest a été réalisé auprès de onze enseignantes et enseignants d'un cégep ne faisant pas partie de l'échantillon.

L'échelle de perception des enseignantes et enseignants par rapport aux mesures offertes par les services adaptés et les services psychosociaux (St-Onge et Tremblay, 2009) comporte trois construits expliquant $63,1 \%$ de la variance, pour un total de 13 énoncés en 4 points allant de pas du tout aidante (1) à tout à fait aidante (4). Le premier construit - l'encadrement, le suivi et l'orientation $(\alpha=0,83)$ - compte six énoncés, dont voici des exemples: «Un encadrement individuel ou psychosocial par un professionnel», «Un encadrement par un pair (tutorat).» Le deuxième construit les mesures habituelles ou traditionnelles offertes $(\alpha=0,84)$ - compte quatre énoncés, par exemple: «La passation des examens dans un local extérieur à la salle de classe», «Le temps supplémentaire pour faire les examens.» Le troisième construit - l'autorisation d'incomplets ou de modifications à l'horaire $(\alpha=0,77)$ - compte trois énoncés.

L'échelle des mesures offertes par les enseignantes et enseignants pour aider les étudiantes et étudiants ayant un TM (St-Onge et Tremblay, 2009) évalue la fréquence à laquelle les enseignantes et enseignants leur ont offert des mesures dans leur classe. Cette échelle constitue un facteur unique expliquant $34,4 \%$ de la variance, pour un total de 13 énoncés en 4 points allant de jamais (1) à souvent (4). Des exemples de mesures sont: «Encadrer un étudiant individuellement »; «Accorder un délai pour un travail.» L'échelle présente une très bonne cohérence interne $(\alpha=0,85)$.

7. L'incomplet temporaire remplace la mention «échec» au bulletin, de sorte que la cote de rendement collégiale n'est pas affectée. L'étudiant ou l'étudiante se voit donner un délai pour compléter les évaluations relatives à son cours. L'incomplet permanent remplace également la mention "échec» au bulletin. Le cas échéant, le cours doit être repris en entier. 
L'échelle des besoins des enseignantes et des enseignants (St-Onge et Tremblay, 2009) mesure le degré auquel ils ressentent différents besoins. Cette échelle comporte deux construits distincts expliquant $48,7 \%$ de la variance, pour un total de 22 énoncés en 4 points allant de pas du tout (1) à tout à fait (4). Le premier construit - le besoin d'être consultés et d'être reconnus $(\alpha=0,89)$ - compte 13 énoncés, dont voici des exemples: «Discuter avec d'autres collègues des difficultés rencontrées en classe avec un étudiant», "Être consultés sur des mesures à mettre en place lorsqu'une problématique se présente dans leur classe.» Le second construit - le besoin d'information et de sensibilisation $(\alpha=0,88)$ - compte neuf énoncés, par exemple: "Être sensibilisés aux troubles mentaux», "Être informés des meilleures façons de faire avec ces étudiants. ${ }^{8}$

Un questionnaire sociodémographique porte sur les variables suivantes: sexe, âge, nombre d'heures travaillées par semaine, statut d'emploi (temporaire ou permanent), nombre d'années d'enseignement, degré de scolarité. La durée de passation du questionnaire final, en anglais et en français, est d'environ 40 minutes.

\section{L'approbation éthique}

Ce projet a été approuvé par le comité d'éthique de la recherche de l'IRDPQ, institut universitaire de réadaptation, auquel la première auteure était rattachée au moment de l'étude, et par un cégep possédant son propre comité d'éthique.

\section{Les analyses statistiques}

Des ANOVA à plan simple ont été effectuées afin d'évaluer s'il existe des différences par rapport aux variables à l'étude (c'est-à-dire les construits des cinq échelles utilisées) quant à certaines caractéristiques: sexe, âge, expérience en enseignement au cégep, source de connaissances sur les TSM. Pour les variables de plus de deux catégories - âge et expérience en enseignement - un test a posteriori de Tukey a été effectué. Seuls les résultats significatifs sont présentés.

\section{Les résultats}

\section{Les participantes et participants}

Un total de 373 enseignantes et enseignants ont répondu au questionnaire, ce qui représente un taux de réponse de $10 \%$. Les questionnaires ont été remplis entièrement (232) ou partiellement (119). Les enseignantes et enseignants qui ont répondu à l'échelle de familiarité ${ }^{9}$ par rapport aux TSM et qui ont abandonné par la suite $(\mathrm{n}=83$, moyenne $=16,8)$ connaissent significativement moins les TSM que ceux qui ont terminé le questionnaire $(\mathrm{n}=232$; moyenne $=18,0), F(1,371)=4,76, p<0,05$.

8. Les questionnaires, anglais et français, sont disponibles en s'adressant à la première auteure.

9. L'échelle de familiarité par rapport aux troubles mentaux a été construite à partir de la question : "Dans quelle mesure êtes-vous familier avec les troubles mentaux suivants?». Par exemple, la dépression, l'anxiété généralisée, le trouble panique, la paranoïa, etc. Cette échelle compte 9 énoncés en 4 points allant de pas du tout familier (1) à tout à fait familier (4).» 
Leur abandon pourrait s'expliquer par un manque d'intérêt vis-à-vis du sujet de la recherche. Pour cette raison, seuls les 232 questionnaires remplis entièrement ont été conservés.

\section{Les données sociodémographiques}

Le tableau 1 présente les données sociodémographiques du personnel enseignant. La majorité des répondantes et des répondants sont des femmes $(72,0 \%)$ entre 39 et 58 ans $(65,9 \%)$ qui travaillent à temps plein $(83,6 \%)$ et qui ont un statut d'emploi permanent $(69 \%)$. Les répondants enseignent au cégep depuis 5 ans et moins $(22,4 \%), 6$ à 15 ans $(35,3 \%)$ et 16 ans ou plus $(42,2 \%)$. Une majorité d'entre eux ont terminé des études universitaires de premier (44,4\%) ou de deuxième cycle $(42,7 \%)$.

Tableau 1. Caractéristiques sociodémographiques des enseignantes et enseignants ( $n=232)$

\begin{tabular}{|c|c|c|c|}
\hline & & $\mathrm{N}$ & $\%$ \\
\hline \multirow[t]{2}{*}{ Sexe } & Femme & 167 & 72,0 \\
\hline & Homme & 65 & 28,0 \\
\hline \multirow[t]{7}{*}{ Âge } & $18-25$ ans & 2 & 0,9 \\
\hline & $26-31$ ans & 20 & 8,6 \\
\hline & $32-38$ ans & 44 & 19,0 \\
\hline & $39-45$ ans & 56 & 24,1 \\
\hline & $46-51$ ans & 43 & 18,5 \\
\hline & $52-58$ ans & 54 & 23,3 \\
\hline & 59 ans et plus & 13 & 5,6 \\
\hline \multirow{6}{*}{$\begin{array}{l}\text { Nombre d'années } \\
\text { d'enseignement au cégep }\end{array}$} & Moins d'un an & 6 & 2,6 \\
\hline & Entre 1 et 5 ans & 46 & 19,8 \\
\hline & Entre 6 et 10 ans & 39 & 16,8 \\
\hline & Entre 11 et 15 ans & 43 & 18,5 \\
\hline & Entre 16 et 20 ans & 40 & 17,2 \\
\hline & Plus de 20 ans & 58 & 25,0 \\
\hline
\end{tabular}

\section{Les analyses univariées}

En ce qui a trait au sexe (Tableau 2), les enseignantes offrent davantage de mesures dans leurs classes aux étudiantes et étudiants présentant un TSM par rapport aux enseignants, $F(1,230)=6,98, p<0,01, \eta^{2}=0,029^{10}$. De plus, elles perçoivent certaines mesures offertes par les services adaptés et les services psychosociaux, dont les mesures d'encadrement, de suivi et d'orientation, comme plus aidantes que ne le croient les hommes, $F(1,230)=4,64, p<0,05, \eta^{2}=0,020$, ainsi que l'autorisation

10. L'état carré $\left(\eta^{2}\right)$ est la variance intergroupes divisée par la variance totale. Il s'agit de la proportion de la variable dépendante expliquée par la variable indépendante. 
d'incomplets ou de modifications à l'horaire de cours, $F(1,230)=7,91, p<0,01$, $\eta^{2}=0,033$. Les enseignantes ressentent davantage le besoin d'être consultées et reconnues quant aux tâches supplémentaires liées à la présence d'étudiantes et d'étudiants présentant un TSM dans leurs classes, $F(1,230)=16,69, p<0,001$, $\eta^{2}=0,068$. Finalement, elles ressentent davantage le besoin d'être informées et sensibilisées par rapport aux différents TSM, $F(1,230)=6,82, p<0,05, \eta^{2}=0,029$.

Tableau 2. ANOVA associées aux attitudes selon le sexe $(\mathbf{n}=\mathbf{2 3 2})$

\begin{tabular}{|l|l|l|l|l|}
\cline { 2 - 5 } \multicolumn{4}{l|}{} & \multicolumn{3}{l|}{ Les mesures offertes } & CM & SC \\
\hline Source & DI & SC & 403,60 & 6,98 \\
\hline Sexe & 1 & 403,60 & 57,843 & \\
\hline Erreur & 230 & 13303,88 & & \\
\hline Total & 231 & 13707,48 & & \\
\hline
\end{tabular}

\begin{tabular}{|l|l|l|l|l|}
\cline { 2 - 5 } \multicolumn{1}{c|}{} & \multicolumn{4}{l}{$\begin{array}{l}\text { La perception du caractère aidant de l'encadrement, du suivi et de I'orientation } \\
\text { vers des services à I'interne ou à I'externe }\end{array}$} \\
\hline Source & DI & SC & CM & F \\
\hline Sexe & 1 & 49,90 & 49,90 & 4,64 \\
\hline Erreur & 230 & 2471,95 & 10,75 & \\
\hline Total & 231 & 2521,85 & & \\
\hline
\end{tabular}

\begin{tabular}{|l|l|l|l|l|}
\cline { 2 - 5 } \multicolumn{4}{c|}{} & \multicolumn{4}{c|}{$\begin{array}{l}\text { La perception du caractère aidant de l'autorisation d'incomplets ou } \\
\text { de modifications à I'horaire de cours }\end{array}$} \\
\hline Source & DI & SC & CM & F \\
\hline Sexe & 1 & 39,47 & 39,47 & $7,91^{\text {w. }}$ \\
\hline Erreur & 230 & 1147,55 & 4,99 & \\
\hline Total & 231 & 1187,02 & & \\
\hline
\end{tabular}

\begin{tabular}{|l|l|l|l|l|}
\cline { 2 - 5 } \multicolumn{4}{c|}{} & Le besoin d'être consultés, d'être reconnus \\
\hline Source & DI & SC & CM & $F$ \\
\hline Sexe & 1 & 595,72 & 595,72 & 16,69 \\
\hline Erreur & 230 & 8207,31 & 35,68 & \\
\hline Total & 231 & 8803,03 & & \\
\hline
\end{tabular}

\begin{tabular}{|l|l|l|l|l|}
\cline { 2 - 5 } \multicolumn{1}{c|}{} & \multicolumn{4}{l}{ Le besoin d'information et de sensibilisation } \\
\hline Source & DI & SC & CM & $F$ \\
\hline Sexe & 1 & 216,15 & 216,15 & $6,82^{*}$ \\
\hline Erreur & 230 & 7289,71 & 31,69 & \\
\hline Total & 231 & 7505,86 & & \\
\hline
\end{tabular}

$" p<0,05 ; " * p<0,01 ; * * " p<0,001$. 
En ce qui concerne l'âge (Tableau 3), l'analyse de variance révèle qu'il existe une seule différence significative entre les trois groupes, soit celle qui est liée à la perception du caractère aidant des mesures d'encadrement, de suivi et d'orientation des services adaptés et des services psychosociaux, $F(2,229)=5,10, p<0,01, \eta^{2}=0,043$. L'analyse a posteriori montre que le personnel enseignant âgé de 38 ans et moins $(M=21,5, E T=2,56)$ et celui âgé de 39 à 51 ans $(M=21,3, E T=3,43)$ perçoivent ces mesures comme plus aidantes, comparativement aux enseignantes et enseignants âgés de 52 ans et plus $(M=19,9, E T=3,56)$.

Tableau 3. ANOVA associée à la perception des mesures d'encadrement, de suivi et d'orientation selon l'âge $(n=232)$

\begin{tabular}{|l|l|l|l|l|}
\hline Source & DI & SC & CM & $F$ \\
\hline Âge & 2 & 107,56 & 53,78 & $5,10 *$ \\
\hline Erreur & 229 & 2414,29 & 10,54 & \\
\hline Total & 231 & 2521,85 & & \\
\hline
\end{tabular}

$* p<0,01$.

Pour ce qui est de l'expérience en enseignement au cégep (Tableau 4), l'analyse de variance révèle une différence quant au nombre de mesures offertes aux étudiantes et étudiants présentant un TSM, $F(2,229)=8,98, p<0,001, \eta^{2}=0,073$. L'analyse a posteriori montre que le personnel enseignant ayant de 6 à 15 années d'expérience ( $M=35,6, E T=7,17)$ ou 16 années d'expérience et plus $(M=35,2$, $E T=6,89)$ offre plus de mesures dans leurs classes que les enseignantes et enseignants ayant 5 années d'expérience et moins $(M=30,6, E T=8,80)$.

Tableau 4. ANOVA associée aux mesures offertes en classe par les enseignantes et les enseignants selon l'expérience en enseignement $(n=232)$

\begin{tabular}{|l|l|l|l|l|}
\hline Source & DI & SC & $C M$ & $F$ \\
\hline Expérience & 2 & 997,22 & 498,61 & $8,98 *$ \\
\hline Erreur & 229 & 12710,26 & 55,50 & \\
\hline Total & 231 & 13707,49 & & \\
\hline$p<0,001$. &
\end{tabular}

Les enseignantes et enseignants qui ont acquis des connaissances sur les TSM lors de leur formation universitaire (Tableau 5), en comparaison du personnel enseignant n'ayant pas reçu une telle formation, ont une meilleure capacité de détection des TSM, $F(1,230)=7,71, p<0,01, \eta^{2}=0,032$. Ils ont davantage confiance en leur capacité à convaincre une étudiante ou un étudiant d'aller chercher de l'aide, 
$F(1,230)=26,73, p<0,001, \eta^{2}=0,104$, et possèdent plus de connaissances et une meilleure compréhension des TSM, $F(1,230)=57,78, p<0,001, \eta^{2}=0,201$. De plus, ils perçoivent l'autorisation d'incomplets ou de modifications à l'horaire de cours comme plus aidante, $F(1,230)=12,68, p<0,001, \eta^{2}=0,052$. Finalement, ces enseignantes et enseignants ressentent moins le besoin d'être informés et sensibilisés par rapport à ces troubles que le personnel enseignant n'ayant pas reçu une telle formation, $F(1,230)=4,24, p<0,05, \eta^{2}=0,018$.

Tableau 5. ANOVA associées aux attitudes selon l'acquisition de connaissances sur les TSM lors d'une formation en milieu universitaire $(n=232)$

\begin{tabular}{|l|l|l|l|l|}
\cline { 2 - 5 } \multicolumn{1}{c|}{} & \multicolumn{4}{l|}{ La capacité de détection d'un trouble mental } \\
\hline Source & DI & SC & CM & F \\
\hline Source de connaissances & 1 & 106,19 & 106,19 & $7,71 \%$ \\
\hline Erreur & 230 & 3168,53 & 13,78 & \\
\hline Total & 231 & 3274,72 & & \\
\hline
\end{tabular}

\begin{tabular}{|l|l|l|l|l|}
\cline { 2 - 5 } \multicolumn{1}{c|}{} & \multicolumn{4}{c|}{ La confiance en sa capacité à convaincre un étudiant d'aller chercher de l'aide } \\
\hline Source & DI & SC & CM & F \\
\hline Source de connaissances & 1 & 226,23 & 226,23 & $26,73^{\text {w.:k }}$ \\
\hline Erreur & 230 & 1946,99 & 8,47 & \\
\hline Total & 231 & 2173,22 & & \\
\hline
\end{tabular}

\begin{tabular}{|l|l|l|l|l|}
\cline { 2 - 5 } \multicolumn{1}{c|}{} & \multicolumn{4}{c|}{ La connaissance et la compréhension des troubles } \\
\hline Source & DI & SC & CM & F \\
\hline Source de connaissances & 1 & 338,04 & 338,04 & $57,78^{\text {w:* }}$ \\
\hline Erreur & 230 & 1345,73 & 5,85 & \\
\hline Total & 231 & 1683,78 & & \\
\hline
\end{tabular}

\begin{tabular}{|l|l|l|l|l|}
\cline { 2 - 5 } \multicolumn{1}{c|}{} & \multicolumn{4}{l|}{$\begin{array}{l}\text { La perception du caractère aidant de l'autorisation d'incomplets ou } \\
\text { de modifications à l'horaire de cours }\end{array}$} \\
\hline Source & DI & SC & CM & F \\
\hline Source de connaissances & 1 & 62,01 & 62,01 & $12,68^{\text {*n: }}$ \\
\hline Erreur & 230 & 1125,01 & 4,89 & \\
\hline Total & 231 & 1187,02 & & \\
\hline
\end{tabular}

\begin{tabular}{|l|l|l|l|l|}
\cline { 2 - 5 } \multicolumn{1}{c|}{} & \multicolumn{4}{l|}{ Le besoin d'information et de sensibilisation } \\
\hline Source & DI & SC & CM & $F$ \\
\hline Source de connaissances & 1 & 135,90 & 135,90 & $4,24 *$ \\
\hline Erreur & 230 & 7369,96 & 32,04 & \\
\hline Total & 231 & 7505,86 & & \\
\hline
\end{tabular}

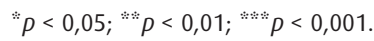


Les enseignantes et enseignants qui ont acquis des connaissances sur les TSM lors d'une formation en milieu de travail (Tableau 6), en comparaison du personnel enseignant n'ayant pas suivi une telle formation, ont davantage confiance en leur capacité à convaincre une étudiante ou un étudiant d'aller chercher de l'aide, $F(1,230)=18,38, p<0,001, \eta^{2}=0,074$. Tout comme ceux et celles ayant acquis des connaissances en milieu universitaire, ils ont davantage de connaissances et une meilleure compréhension des TSM, $F(1,230)=28,28, p<0,001, \eta^{2}=0$,109. Ils perçoivent l'autorisation d'incomplets ou de modifications à l'horaire de cours comme plus aidante, $F(1,230)=7,16, p<0,01, \eta^{2}=0,030$, et ressentent moins le besoin d'être informés et sensibilisés par rapport aux TSM que le personnel enseignant n'ayant pas reçu une formation en milieu de travail, $F(1,230)=4,89, p<0,05, \eta^{2}=0,021$.

\section{Tableau 6. CANOVA associées aux attitudes selon l'acquisition de connaissances sur} les TSM lors d'une formation en milieu de travail $(n=232)$

\begin{tabular}{|l|l|l|l|l|}
\cline { 2 - 5 } \multicolumn{1}{c|}{} & \multicolumn{4}{c|}{ La confiance en sa capacité à convaincre un étudiant d'aller chercher de l'aide } \\
\hline Source & DI & SC & CM & F \\
\hline Source de connaissances & 1 & 160,79 & 160,79 & 18,38 \\
\hline Erreur & 230 & 2012,44 & 8,75 & \\
\hline Total & 231 & 2173,22 & & \\
\hline
\end{tabular}

\begin{tabular}{|l|l|l|l|l|}
\cline { 2 - 5 } \multicolumn{1}{c|}{} & \multicolumn{4}{l|}{ La connaissance et la compréhension des troubles } \\
\hline Source & DI & SC & CM & F \\
\hline Source de connaissances & 1 & 184,11 & 184,11 & 28,28 \\
\hline Erreur & 230 & 1499,66 & 6,52 & \\
\hline Total & 231 & 1683,78 & & \\
\hline
\end{tabular}

\begin{tabular}{|l|l|l|l|l|}
\cline { 2 - 5 } \multicolumn{1}{c|}{} & \multicolumn{4}{l}{$\begin{array}{l}\text { La perception du caractère aidant de l'autorisation d'incomplets ou } \\
\text { de modifications à I'horaire de cours }\end{array}$} \\
\hline Source & DI & SC & CM & F \\
\hline Source de connaissances & 1 & 35,69 & 35,62 & $7,16^{\text {*n: }}$ \\
\hline Erreur & 230 & 1151,40 & 5,01 & \\
\hline Total & 231 & 1187,02 & & \\
\hline
\end{tabular}

\begin{tabular}{|l|l|l|l|l|}
\cline { 2 - 5 } \multicolumn{1}{c|}{} & \multicolumn{4}{l|}{ Le besoin d'information et de sensibilisation } \\
\hline Source & DI & SC & CM & F \\
\hline Source de connaissances & 1 & 156,14 & 156,14 & 4,89 \\
\hline Erreur & 230 & 7349,71 & 31,96 & \\
\hline Total & 231 & 7505,86 & & \\
\hline
\end{tabular}

$" p<0,05 ; " * p<0,01 ; " * \cdots=0,001$. 
Les membres du personnel enseignant qui ont acquis des connaissances sur les TSM par l'intermédiaire des médias - télévision, radio, journaux, magazines, livres, Internet - (Tableau 7), par comparaison avec le personnel n'ayant pas acquis ses connaissances par ce moyen, ont davantage d'attitudes négatives à l'égard des TSM, $F(1,230)=5,63, p<0,05, \eta^{2}=0,024$. Ils possèdent également une plus grande capacité à détecter les TSM, $F(1,230)=3,81, p<0,1, \eta^{2}=0,016$.

Tableau 7. ANOVA associées aux attitudes selon l'acquisition de connaissances sur les TM par l'intermédiaire des médias $(\mathbf{n}=\mathbf{2 3 2})$

\begin{tabular}{|l|l|l|l|l|}
\cline { 2 - 5 } \multicolumn{1}{c|}{} & \multicolumn{4}{l|}{ La capacité de détection d'un trouble mental } \\
\hline Source & DI & SC & CM & F \\
\hline Source de connaissances & 1 & 53,35 & 53,35 & $3,81^{*}$ \\
\hline Erreur & 230 & 3221,37 & 14,01 & \\
\hline Total & 231 & 3274,72 & & \\
\hline
\end{tabular}

\begin{tabular}{|l|l|l|l|l|}
\cline { 2 - 5 } \multicolumn{1}{l|}{ Les attitudes négatives à I'égard des TM } \\
\hline Source & DI & SC & CM & F \\
\hline Source de connaissances & 1 & 155,46 & 155,46 & 5,63 \\
\hline Erreur & 230 & 6355,50 & 27,63 & \\
\hline Total & 231 & 6510,96 & & \\
\hline
\end{tabular}

$" p<0,1 ; " p<0,05$.

\section{Discussion}

L'objectif de la présente recherche était de vérifier s'il existait des différences en ce qui a trait aux attitudes du personnel enseignant ainsi qu'à ses besoins par rapport à l'âge, au sexe, à l'expérience en enseignement et aux sources de connaissances sur les TSM.

Dans un premier temps, les femmes offrent davantage de mesures dans leurs classes aux étudiantes et étudiants présentant un TSM. Cela pourrait expliquer pourquoi elles ressentent davantage le besoin d'être consultées et reconnues dans leur travail auprès de ces étudiantes et étudiants ainsi que d'être sensibilisées et informées quant aux TSM. La différence dans l'offre de mesures pourrait prendre racine dans les rôles sexuels typiquement valorisés par la société (macrosystème du MDH$\mathrm{PPH}$ ). Les caractéristiques encouragées chez les hommes font qu'ils tendent à réprimer leurs émotions et à éviter de rechercher de l'aide (Payne, Swami et Stanistreet, 2008). Dans cette optique, il est possible que les enseignants jugent défavorablement les étudiants qui dévient de ces standards sociaux en communiquant leurs symptômes 
psychiatriques. Au contraire, des traits de personnalité plus développés chez les femmes, telle la tendresse (Feingold, 1994), pourraient expliquer que celles-ci offrent davantage d'aide aux étudiantes et étudiants en difficulté.

Les enseignantes et enseignants plus jeunes, par comparaison avec ceux qui sont plus âgés, perçoivent les mesures d'encadrement, de suivi et d'orientation vers les services internes et externes (p. ex. «un encadrement individuel ou psychosocial par un professionnel») comme plus aidantes. Cela semble refléter les changements observés dans la population générale qui reconnaît de plus en plus les bases biologiques des TSM (Schomerus et al., 2012). Une conséquence possible de cette évolution est l'augmentation de la confiance du public dans la capacité des experts de la santé à influer sur le cours des TSM. Ainsi, de nos jours, la population est plus encline à recommander le recours à la médicalisation ou à un psychiatre, et elle tend à encourager davantage la recherche d'aide auprès d'un psychothérapeute (Schomerus et al., 2012). Il est probable que la connaissance accrue des corrélats biologiques des TSM soit attribuable aux individus plus jeunes, dont des enseignantes et des enseignants, qui sont mieux renseignés sur les progrès scientifiques récents en raison de leur formation scolaire. Toutefois, selon Schomerus et ses collègues (2012), cette connaissance ne s'accompagne pas d'une diminution de la stigmatisation et de la discrimination des personnes ayant un TSM. La compréhension des bases biologiques des TSM augmenterait donc l'utilité perçue de l'aide professionnelle, sans améliorer l'attitude générale.

Les enseignantes et enseignants plus expérimentés offrent plus de mesures dans leurs classes, comparativement à ceux qui le sont moins. Au cours de leur carrière, les enseignantes et enseignants entrent en contact avec des étudiantes et des étudiants demandant des mesures d'aide. Ces échanges sont susceptibles d'augmenter leur connaissance de ces mesures et de les sensibiliser quant à leurs responsabilités professionnelles vis-à-vis des étudiantes et étudiants présentant un TSM. Ainsi, l'expérience en enseignement s'accompagnerait d'une croissance de la proactivité dans l'offre de mesures d'aide.

Les enseignantes et enseignants ayant acquis des connaissances sur les TSM durant leur formation universitaire offrent davantage de mesures aux étudiantes et étudiants. Ce comportement positif peut s'expliquer de diverses manières. D'abord, les formations en milieu universitaire sont associées à une meilleure connaissance et compréhension des troubles. Ces connaissances pourraient contrer certains stigmates encore répandus, notamment quant à la dangerosité des individus présentant un TSM (Schomerus et al., 2012). Par ailleurs, cette formation en contexte universitaire est associée à une meilleure capacité de détection des troubles mentaux. Aussi, l'acquisition d'outils d'intervention lors de ces formations pourrait expliquer la confiance accrue des enseignantes et des enseignants en leur capacité à convaincre un étudiant d'aller chercher de l'aide. En somme, ces deux atouts sont susceptibles d'avoir contribué au plus grand nombre de mesures offertes.

Le personnel enseignant ayant suivi une formation en milieu de travail montre également une attitude plus favorable à l'égard des TSM. Ce type de formation a l'avantage d'être court. Depuis la création, en 2012, de la fonction de conseiller ou de 
conseillère de soutien aux services adaptés des collèges, ce type de formation est probablement de plus en plus offert. Dans son enquête, la FNEEQ (2013) mentionne que $71 \%$ de l'ensemble des établissements collégiaux tiennent des rencontres de groupe à chaque session pour échanger avec le personnel enseignant sur les étudiantes et étudiants ayant des besoins particuliers.

Finalement, les enseignantes et enseignants ayant acquis des connaissances sur les TSM par l'entremise des médias ont davantage d'attitudes négatives. Cela est préoccupant, considérant que $88 \%$ des participants de la présente étude rapportent avoir été exposés aux médias. Selon Brockelman et ses collaborateurs (2006), l'exposition aux médias n'est pas associée à la confiance ou à la facilité à œuvrer auprès des étudiantes et étudiants présentant un TSM. Néanmoins, la plupart des enseignantes et enseignants estiment que les médias ont influencé leur perception à l'égard de ces étudiantes et étudiants (Brockelman et al., 2006). Selon Stuart (2006), les médias de divertissement et d'informations télévisuelles présentent une image dramatisée des TSM qui en accentue les aspects de dangerosité et d'imprévisibilité. Ainsi, les auditeurs pourraient développer une vision irréaliste et de l'intolérance à l'égard des individus présentant un TSM (Stuart, 2006). Ce phénomène pourrait également expliquer la plus grande capacité de détection des TSM chez les enseignantes et enseignants exposés aux médias. Ceux-ci étant craintifs à l'égard des étudiantes et des étudiants présentant un TSM, les comportements inhabituels dans la classe seraient davantage interprétés comme étant des manifestations de symptômes psychiatriques.

L'effet de sélectivité et d'amplification des médias peut être contrebalancé par les formations universitaires et celles offertes en milieu de travail. Par exemple, il est possible de confronter la croyance en la dangerosité des individus présentant un TSM. Selon une revue de la documentation (Rüsch, Angermeyer et Corrigan, 2005) et une méta-analyse (Corrigan, Morris, Michaels, Rafacz et Rüsch, 2012), l'éducation notamment la présentation d'informations contradictoires aux stigmates - a un effet positif sur l'attitude et les intentions comportementales vis-à-vis des individus présentant un TSM. Au contraire, renseigner sur les variables biologiques impliquées dans les TSM pourrait augmenter la stigmatisation (Rüsch et al., 2005). Finalement, il n'apparaît pas optimal d'induire «une connaissance pointue de l'ensemble des manifestations [des troubles]. Pour faciliter l'intégration des étudiants en situation de handicap dans les classes et que cette intégration ait le moins d'impact négatif sur les activités d'enseignement, la Commission estime qu'il est préférable que les enseignants puissent, dans une perspective de développement professionnel, bénéficier d'une offre de formation qui soit en lien avec l'acquisition d'un certain nombre de compétences relatives à cette intégration [...]» (CDPDJ, 2012, p. 155). Dans cette optique, il est préférable qu'ils soient informés des meilleures façons de faire avec ces étudiantes et étudiants.

Malgré les bienfaits éprouvés de l'éducation, son efficacité est inférieure au contact avec les individus présentant un TSM (Corrigan et al., 2012). Par exemple, ce contact est associé à une diminution de la perception de leur dangerosité (Couture et Penn, 2003). Il est donc nécessaire que le personnel enseignant puisse entrer en relation avec les étudiantes et étudiants présentant des symptômes psychiatriques. À cet 
égard, une pratique défavorable a été constatée lors de cette étude. Certains services adaptés des collèges participants possédaient une "liste noire» d'enseignantes et d'enseignants présentant une attitude négative face aux TSM. Ils s'assuraient ainsi que les étudiantes et étudiants ayant un tel trouble ne soient pas inscrits dans leurs classes. Malheureusement, cette pratique d'évitement vise à limiter le contact, favorisant le maintien des préjugés et des craintes vis-à-vis de ces étudiantes et étudiants.

Le faible taux de participation est une limite de cette étude. Il est possible que les enseignantes et enseignants ayant répondu au sondage partagent un intérêt à l'égard des TSM. Toutefois, la variabilité dans les réponses semble indiquer que cet effet est limité. Par ailleurs, bien que trois variables dépendantes n'étaient pas distribuées normalement, l'ANOVA est robuste à la non-normalité de ce type de variables (Schmider et al., 2010). Finalement, nous n'avons pas appliqué de correction lors des analyses de variance, augmentant le risque de commettre une erreur de type 1.

En conclusion, étant donné le rôle central que semble jouer la formation en contexte universitaire, mais aussi en contexte de travail, sur les attitudes favorables des enseignants et des enseignants à l'égard des étudiantes et des étudiants ayant un TSM, il serait important qu'une formation sur les TSM soit accessible dans les cursus universitaires, principalement dans les disciplines en éducation, mais aussi dans les autres disciplines enseignées au secteur collégial. À la lumière de nos résultats, il serait également indiqué de traiter de l'impact négatif des médias comme source de connaissances sur les TSM. Enfin, le recours au MDH-PPH comme source d'analyse conceptuelle du contexte scolaire peut nous aider à mieux comprendre les éléments contributifs à la persévérance scolaire des étudiants ayant un TSM.

\section{Références bibliographiques}

ABRIC, J.-C. (2011). Pratiques sociales et représentations. Paris: Presses universitaires de France.

ABU-HAMOUR, B. (2013). Faculty attitudes toward students with disabilities in a public university in Jordan. International Education Studies, 6(12), 74-81.

BECKER, M., MARTIN, L., WAJEEH, E., WARD, J. et SHERN, D. (2002). Students with mental illnesses in a university setting. Faculty and student attitudes, beliefs, knowledge, and experiences. Psychiatric Rehabilitation Journal, 25(4), 359-368.

BROCKELMAN, K. F., CHADSEY, J. G. et LOEB, J. W. (2006). Faculty perceptions of university students with psychiatric disabilities. Psychiatric Rehabilitation Journal, 30(1), 23-30. 
COMMISSION DES DROITS DE LA PERSONNE ET DES DROITS DE LA JEUNESSE. (2012). L'accommodement des étudiants et étudiantes en situation de handicap dans les établissements d'enseignement collégial. Récupéré de http://www.cdpdj.qc.ca/publications/accommodement_handicap_collegial.pdf

CORRIGAN, P. W., MORRIS, S. B., MICHAELS, P. J., RAFACZ, J. D. et RÜSCH, N. (2012). Challenging the public stigma of mental illness. A meta-analysis of outcome studies. Psychiatric Services, 63(10), 963-973.

COUTURE, S. M. et PENN, D. L. (2003). Interpersonal contact and the stigma of mental illness: A review of the literature. Journal of Mental Health, 12(2), 291-305.

FÉDÉRATION NATIONALE DES ENSEIGNANTES ET DES ENSEIGNANTS DU QUÉBEC (FNEEQ). (2013). La présence de la population étudiante ayant des besoins particuliers et son impact sur la charge d'enseignement. Rapport des travaux du Comité national de rencontre. Personnel enseignant.

FEINGOLD, A. (1994). Gender differences in personality: A meta-analysis. Psychological Bulletin, 116(3), 429-456.

FOUGEYROLLAS, P., CLOUTIER, R., BERGERON, H., CÔTÉ, J. et ST-MICHEL, G. (1998). Classification québécoise. Processus de production du handicap. LacSaint-Charles, Québec: Réseau international sur le Processus de production du handicap (RIPPH).

FOUGEYROLLAS, P. (2010). La funambule, le fil et la toile. Transformations réciproques du sens du handicap. Québec: Presses de l'Université Laval.

HADDOCK, G. et ZANNA, M. P. (1993). Predicting prejudicial attitudes: The importance of affect, cognition and the feeling-belief dimension. Dans L. McAlister et M. L. Rothschild (dir.), Advances in Consumer Research (vol. 20, p. 315-318). Provo, UT: Association for Consumer Research.

HAMMEL, C. A. (2010). Faculty knowledge of disability law and its relationship to attitude, education and experience (Thèse de doctorat). Accessible par ProQuest Dissertations \& Theses (3383886).

HAWKINS, D. F. (2013). The attitudes of college faculty toward students with disabilities at a church-affiliated institution (Thèse de doctorat). Accessible par ProQuest Dissertations \& Theses (3519052).

KRASKA, M. (2003). Postsecondary students with disabilities and perceptions of faculty members. Journal for Vocational Special Needs Education, 25(2), 11-19.

LEYSER, Y. et GREENBERGER, L. (2008). College students with disabilities in teacher education: Faculty attitudes and practices. European Journal of Special Needs Education, 23(3), 237-251.

LOMBARDI, A. (2010). Measuring faculty attitudes and perceptions toward disability at a four-year university: A validity study (Thèse de doctorat). Accessible par ProQuest Dissertations \& Theses (3407262). 
MURRAY, C., LOMBARDI, A., WREN, C. T. et KEYS, C. (2009). Associations between prior disability-focused training and disability-related attitudes and perceptions among university faculty. Learning Disability Quarterly, 32(2), $87-100$

MURRAY, C., WREN, C. T. et KEYS, C. (2008). University faculty perceptions of students with learning disabilities: Correlates and group differences. Learning Disability Quarterly, 31(3), 95-113.

PAYNE, S., SWAMI, V. et STANISTREET, D. L. (2008). The social construction of gender and its influence on suicide: A review of the literature. Journal of Men's Health \& Gender, 5(1), 23-35.

RAO, S. (2004). Faculty attitudes and students with disabilities in higher education: A literature review. College Student Journal, 38(2), 191-198.

RÜSCH, N., ANGERMEYER, M. C. et CORRIGAN, P. W. (2005). Mental illness stigma: Concepts, consequences, and initiatives to reduce stigma. European Psychiatry, 20(8), 529-539.

SCHOMERUS, G., SCHWAHN, C., HOLZINGER, A., CORRIGAN, P. W., GRABE, H. J., CARTA, M. G. et ANGERMEYER, M. C. (2012). Evolution of public attitudes about mental illness: A systematic review and meta-analysis. Acta Psychiatrica Scandinavica, 125(6), 440-452.

SCHMIDER, E., ZIEGLER, M., DANAY, E., BEYER, L. et BÜHNER, M. (2010). Is it really robust? Reinvestigating the robustness of ANOVA against violations of the normal distribution assumption. European Journal of Research Methods for the Behavioral and Social Sciences, 6(4), 147-151.

ST-ONGE, M. et TREMBLAY, J. (2009). L'offre de services pour les étudiants et étudiantes des cégeps ayant un problème de santé mentale ou un trouble mental. Les analyses de validité de construit et les statistiques descriptives des échelles. Québec, Université Laval, École de service social.

ST-ONGE, M., TREMBLAY, J. et GARNEAU, D. (2009). L'offre de services pour les étudiants et étudiantes des cégeps ayant un problème de santé mentale ou un trouble mental. Rapport final de recherche. Québec, Université Laval, École de service social.

STUART, H. (2006). Media portrayal of mental illness and its treatments: What effect does it have on people with mental illness? CNS Drugs, 20(2), 99-106.

SWARTZ, H. A., FRANK, E. et FRANKEL, D. (2008). Psychothérapie interpersonnelle et des rythmes sociaux (PTIRS) dans le trouble bipolaire II : structure du traitement et exemples cliniques. Santé mentale au Québec, XXXIII(2), 151-184.

WORTHY, K. L. (2014). Faculty attitudes toward students with disabilities at a Southern HBCU (Thèse de doctorat). Accessible par ProQuest Dissertations \& Theses (3587522). 